\title{
FULL-WAVEFORM INVERSION FOR THE RECONSTRUCTION OF ELECTROMAGNETIC PROPERTY PROFILES USING PLANE MICROWAVES
}

\author{
Namho Joh ${ }^{1}$ and Jun Won Kang ${ }^{1}$ \\ ${ }^{1}$ Department of Civil Engineering, Hongik University \\ Wausan-ro 94, Mapo-gu, Seoul 04066, Republic of Korea \\ e-mail: johst99@ naver.com, jwkang@hongik.ac.kr
}

Keywords: Perfectly-Matched-Layers (PMLs), Plane electromagnetic waves, Lagrangian, Full-waveform Inversion, Maxwell's equations.

\begin{abstract}
This study is concerned with microwave full-waveform inversion method for the reconstruction of electromagnetic properties in heterogeneous one-dimensional media. Perfectly-Matched-Layers (PMLs) are introduced as wave-absorbing boundary to reduce the originally infinite medium to a finite computational domain. The microwave full-waveform inversion method is based on an optimization approach constrained by PML-endowed Maxwell's equations for plane electromagnetic waves. The approach attempts to minimize the objective functional consisting of the $L^{2}$ norm of a misfit between calculated and observed electric fields. Specifically, Lagrangian was constructed comprising the objective functional augmented by the PML-endowed Maxwell's equations via Lagrange multipliers. Enforcing stationarity of the Lagrangian yields time-dependent state, adjoint, and time-independent control problems, which can be iteratively solved to update the electromagnetic properties of the medium such as permittivity and permeability. We used Fletcher-Reeves conjugate gradient method for updating the electromagnetic property profiles, and explored Tikhonov and Total Variation regularization methods to relieve the ill-posedness inherent in the fullwaveform inversion. Numerical results are reported showing the successful reconstruction of both smooth and sharply-varying electromagnetic property profiles with appropriate regularization methods.
\end{abstract}

\section{INTRODUCTION}

In the last several decades, microwave nondestructive evaluation (NDE) of civil structures has been paid considerable attention by both academics and industry owing to the advantages such as low cost, good penetration in dielectric materials, good resolution, and contactless feature of sensors. The method has been found to be potentially excellent in detecting cracks, voids, flaws, defects, moisture contents, layers, etc. of composite structures by itself or with the combination with other NDE methods. Recent applications of the method includes, but not limited to, damage exploration of FRP sheets [1], quality control and condition assessment of reinforced concrete structures [2,3]. 
Microwave NDE methods generally use electromagnetic waves with frequencies between $300 \mathrm{MHz}$ and $100 \mathrm{GHz}$ to inspect and characterize structures and materials. For dielectric materials, microwaves may have wavelengths of orders of centimeters to millimeters, and it has been shown that cracks and layer thickness of heterogeneous dielectric structures could be measured using microwaves of such wavelengths [4,5]. However, microwave NDE methods have been more implemented in academic research environment, rather than for practical applications so far. One of the reasons for this is probably that the microwave NDE technique and the interpretation of test data are fairly complicated. Despite the difficulties and limited availability of commercial microwave NDE systems, microwave NDE methods have achieved great progress in the development of sophisticated inversion procedure and microwave imaging system for structure monitoring and biomedical applications. In particular, recent development of innovative inverse scattering methods with various optimization algorithms deserves close attention [6,7].

This work seeks the development of a microwave inverse scattering method for subsurface imaging of structures. The proposed inversion method is based on using full waveforms rather than using the waveform of specific frequencies. This kind of method is called full-waveform inversion method, and it has been shown to be effective in unveiling unknown material properties in seismic imaging and geophysical probing applications, etc. [8,9]

The target for reconstruction in this work is the spatial distribution of permittivity and permeability profiles of heterogeneous media. To this end, an optimization approach constrained by Maxwell's equations for plane electromagnetic waves was introduced. The approach attempts to minimize the objective functional consisting of the $L^{2}$ norm of a misfit between calculated and measured electric fields. Specifically, Lagrangian was constructed comprising the objective functional augmented by the Maxwell's equations. Enforcing stationarity of the Lagrangian yields time-dependent state, adjoint, and time-independent control problems, which can be solved iteratively to update the permittivity and permeability profiles of the medium. To satisfy far-field radiation condition of electromagnetic waves, Perfectly-Matched-Layers (PMLs) were introduced as wave-absorbing boundaries next to the finite computational domain. Formulation and numerical examples of plane microwave inversion are presented in the subsequent Chapters.

\section{FORWARD PROBLEM}

To conduct full-waveform inversion analysis, forward solutions of wave equations are indispensable since the solutions should be utilized in the overall inversion process. In this work, plane microwaves are treated as probing waves for the characterization of material profiles, such that the domain of wave propagation can be modelled as a one-dimensional one. Fig. 1 illustrates the one-dimensional domain truncated by PMLs on both sides. For the forward modelling of plane electromagnetic waves in the PML-truncated domain, modified Maxwell's equations discussed in [10-12] are introduced. Therefore, the forward problem to find electric and magnetic fields in the PML-truncated domain can be formulated as the following: Find $E_{x}=E_{x}(z, t)$ and $H_{y}=H_{y}(z, t)$, such that:

$$
\begin{gathered}
\mu \frac{\partial H_{y}}{\partial t}+c_{\mathrm{ref}} g \mu H_{y}+\frac{\partial E_{x}}{\partial z}=0 \\
\epsilon \frac{\partial^{2} E_{x}}{\partial t^{2}}+c_{\mathrm{ref}} g \epsilon \frac{\partial E_{x}}{\partial t}+\frac{\partial^{2} H_{y}}{\partial z \partial t}=-\frac{\partial J}{\partial t}-c_{\mathrm{ref}} g J
\end{gathered}
$$

and 


$$
\begin{gathered}
E_{x}\left(-L_{t l}, t\right)=E_{x}\left(L_{t r}, t\right)=0 \\
H_{y}\left(-L_{t l}, t\right)=H_{y}\left(L_{t r}, t\right)=0 \\
E_{x}(z, 0)=\frac{\partial E_{x}}{\partial t}(z, 0)=0 \\
H_{y}(z, 0)=0
\end{gathered}
$$

where $E_{\mathrm{x}}(z, t)$ and $H_{\mathrm{y}}(z, t)$ are the electric and magnetic fields polarized in the $x$ and $y$ directions, respectively. $J$ is current density, and $\epsilon\left(=\epsilon_{0} \epsilon_{r}\right)$ and $\mu\left(=\mu_{0} \mu_{r}\right)$ denote the permittivity and permeability of the medium, respectively. $c_{\text {ref }}\left(=1 / \sqrt{\epsilon_{0} \mu_{0}}\right)$ denotes reference wave velocity, which was taken as the velocity of electromagnetic waves in free space. $g(z)$ represents an attenuation function that enforces amplitude decay of waves within the PML. The value of the attenuation function $g(z)$ is zero within the regular domain $\left(-L_{l} \leq z \leq L_{\mathrm{r}}\right)$, whereas $g(z)>0$ within the PML $\left(-L_{t l} \leq z \leq-L_{l}\right.$ and $\left.L_{r} \leq z \leq L_{t r}\right)$.

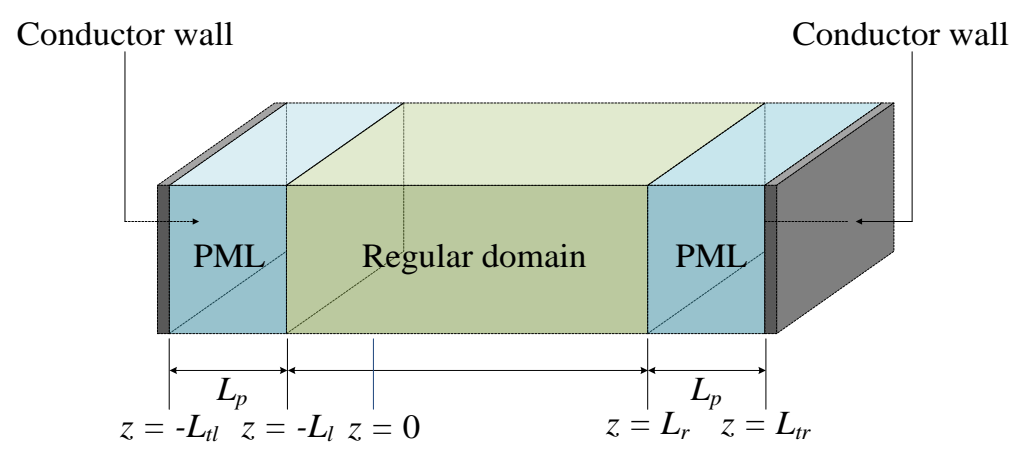

Figure 1: schematics of the PML-truncated domain

\section{INVERSE PROBLEM}

\subsection{PDE-constrained optimization}

The inverse medium problem of interest here is to reconstruct the permittivity profile of heterogeneous medium surrounded by PMLs, given measured electric fields to prescribed excitation at a point in the domain. The inverse problem can be formulated as the following optimization problem constrained by governing partial differential equations:

$$
J:=\frac{1}{2} \sum_{i=1}^{N_{r}} \int_{-L_{t l}}^{L_{t r}} \int_{0}^{T}\left[E_{x}\left(z_{i}, t\right)-E_{m}\left(z_{i}, t\right)\right]^{2} \delta\left(z-z_{i}\right) d t d z+R(\epsilon)
$$

subject to Eqs. (1)-(2).

The objective functional $J$ in Eq. (3) consists of a misfit functional represented as the $L^{2}$ norm of difference between measured electric field $E_{m}\left(z_{i}, t\right)$ and calculated electric field $E_{x}\left(z_{i}, t\right) . N_{r}$ denotes the number of receivers, and $z_{i}$ is the location of receivers. To alleviate the ill-posedness of the inverse problem, regularization term $R(\epsilon)$ was introduced as a function of permittivity to be recovered. In this work, Tikhonov (TN) and Total Variation (TV) regularization schemes were used to investigate the regularization effect on the reconstructed permittivity profile of the PML-truncated domain. If TN regularization scheme 
is used, $R(\epsilon)$ can take the form:

$$
R^{T N}(\epsilon):=\frac{r_{\epsilon}}{2} \int_{L_{t l}}^{L_{t r}}\left(\frac{d \epsilon}{d z}\right)^{2} d z
$$

whereas if TV regularization scheme is used, $R(\epsilon)$ can be formulated as:

$$
R^{T V}(\epsilon):=r_{\epsilon} \int_{L_{t l}}^{L_{t r}}\left[\left(\frac{d \epsilon}{d z}\right)^{2}+\beta\right] d z
$$

where $r_{\epsilon}$ is a regularization factor controlling the amount of penalty on the gradient of permittivity. In Eq. (5), a small parameter $\beta$ was included to make $R^{T V}(\epsilon)$ differentiable when $d \epsilon / d z=0$. It has been known that $\mathrm{TN}$ scheme is suited for the reconstruction of smooth profiles, whereas TV scheme is more relevant for the reconstruction of sharplyvarying target profiles.

\subsection{Lagrangian functional}

To solve the PDE-constrained optimization problem, augmented Lagrangian method was introduced, by which the constrained optimization problem can be replaced by unconstrained optimization problem. The objective functional in Eq. (3) was augmented by side-imposing the PML-endowed Maxwell's equations via Lagrange multipliers to form the following Lagrangian functional.

$$
\begin{aligned}
\mathcal{L}\left(E_{x},\right. & \left.H_{y}, \lambda_{E_{x}}, \lambda_{H_{y}}, \epsilon\right) \\
:= & \frac{1}{2} \int_{-L_{t l}}^{L_{t r}} \int_{0}^{T}\left[E_{x}(z, t)-E_{m}(z, t)\right]^{2} \delta(z) d t d z+R_{\varepsilon}(\varepsilon) \\
& +\int_{-L_{t l}}^{L_{t r}} \int_{0}^{T} \lambda_{H_{y}}\left(\mu \frac{\partial H_{y}}{\partial t}+c_{\mathrm{ref}} g \mu H_{y}+\frac{\partial E_{x}}{\partial z}\right) d t d z \\
& +\int_{-L_{t l}}^{L_{t r}} \int_{0}^{T} \lambda_{E_{x}}\left(\epsilon \frac{\partial^{2} E_{x}}{\partial t^{2}}+c_{\mathrm{ref}} g \epsilon \frac{\partial E_{x}}{\partial t}+\frac{\partial^{2} H_{y}}{\partial z \partial t}=-\frac{\partial J}{\partial t}-c_{\mathrm{ref}} g J\right) d t d z
\end{aligned}
$$

The permittivity profile $\epsilon(z)$ is sought to be reconstructed in an attempt to minimize the Lagrangian functional. The boundary and initial conditions in Eqs. (2a)-(2d) are explicitly imposed in the inversion process.

\subsection{First-order optimality conditions}

Optimal solutions of the state $\left(E_{x}, H_{y}\right)$, adjoint $\left(\lambda_{E_{x}}, \lambda_{H_{y}}\right)$, and control $(\epsilon)$ variables of the Lagrangian functional can be sought by enforcing the first variations of the Lagrangian to vanish. First of all, enforcing the vanishing of the first variations of $\mathcal{L}$ with respect to the Lagrange multipliers $\lambda_{E_{x}}$ and $\lambda_{H_{y}}$ results in the system of equations identical to Eqs. (1a) and (1b) for the forward problem.

Second, enforcing the vanishing of the first variations of $\mathcal{L}$ with respect to the state variables $E_{x}$ and $H_{y}$ yields the following mixed adjoint problem: Find $\lambda_{E_{x}}=\lambda_{E_{x}}(z, t)$ and $\lambda_{H y}=\lambda_{H y}(z, t)$, such that:

$$
\epsilon \frac{\partial^{2} \lambda_{E_{x}}}{\partial t^{2}}-c_{\mathrm{ref}} g \epsilon \frac{\partial \lambda_{E_{x}}}{\partial t}-\frac{\partial \lambda_{H_{y}}}{\partial z}=-\left[E_{x}\left(z_{i}, t\right)-E_{m}\left(z_{i}, t\right)\right] \delta\left(z-z_{i}\right)
$$




$$
\mu \frac{\partial^{2} \lambda_{H_{y}}}{\partial t}-c_{\text {ref }} g \mu \lambda_{H_{y}}-\frac{\partial \lambda_{E_{x}}}{\partial z \partial t}=0
$$

and

$$
\begin{gathered}
\lambda_{E_{x}}(z, T)=\frac{\partial \lambda_{E_{x}}}{\partial t}(z, T)=0 \\
\lambda_{H_{y}}(z, T)=0
\end{gathered}
$$

The adjoint equations (7a) and (7b) are also PML-endowed, and coupled in terms of $\lambda_{E_{x}}$ and $\lambda_{H y}$. The source term of the adjoint problem depends on the misfit between computed and measured electric fields as indicated in Eq. (7a). It is noted that the adjoint problem is a final value problem, as opposed to the initial-value state problem.

Third, enforcing the vanishing of the first variations of $\mathcal{L}$ with respect to the control variable $\epsilon$ entails the following time-invariant control problem: Find $\epsilon=\epsilon(z)$, such that:

$$
\begin{array}{r}
-R_{\epsilon} \frac{\partial^{2} \epsilon}{\partial z^{2}}+\int_{0}^{T}\left(\lambda_{E_{x}} \frac{\partial^{2} E_{x}}{\partial t^{2}}+c_{\text {ref }} g \lambda_{E_{x}} \frac{\partial E_{x}}{\partial t}\right) d t=0 \\
\frac{d \epsilon}{d z}\left(-L_{t l}\right)=\frac{d \epsilon}{d z}\left(L_{t r}\right)=0
\end{array}
$$

To reach Eq. (9a), TN regularization scheme was used in Eq. (4). If, instead, TV regularization scheme were used, the control equation would become

$$
-R_{\epsilon} \beta \frac{\partial^{2} \epsilon}{\partial z^{2}}\left[\left(\frac{d \epsilon}{d z}\right)^{2}+\beta\right]^{-\frac{3}{2}}+\int_{0}^{T}\left(\lambda_{E_{x}} \frac{\partial^{2} E_{x}}{\partial t^{2}}+c_{\mathrm{ref}} g \lambda_{E_{x}} \frac{\partial E_{x}}{\partial t}\right) d t=0
$$

The control problem is a boundary-value problem in terms of $\epsilon$, which can be solved once the state and adjoint solutions are obtained.

\section{MATERIAL PROPERTY UPADTE}

The state, adjoint, and control problems can be solved simultaneously to obtain the optimal solutions of the inverse problem. However, the computational cost is expensive and the approach may not be practical for highly ill-posed inverse problems. In this work, a reduced space approach was introduced, where the permittivity profile is updated repeatedly as inversion iteration progresses. At each inversion iteration, the state variables $E_{x}$ and $H_{y}$ are calculated by solving Eqs. (1)-(2) assuming the distribution of permittivity profile within the domain. Then the adjoint variables $\lambda_{E_{x}}$ and $\lambda_{H y}$ are calculated by solving Eqs. (7) using the state solutions obtained earlier. Finally, the control variable $\epsilon$ is updated using the calculated state and adjoint solutions. The permittivity profile at $k$-th inversion iteration can be updated as

$$
\boldsymbol{\epsilon}_{k+1}=\boldsymbol{\epsilon}_{k}+\alpha \boldsymbol{d}_{k}
$$

in which $\alpha$ is step length, and $\boldsymbol{d}_{\boldsymbol{k}}$ is search direction vector. $\boldsymbol{\epsilon}_{\boldsymbol{k}}$ is the vector of nodal permittivity values at $k$-th inversion iteration. The search direction vector $\boldsymbol{d}_{\boldsymbol{k}}$ was determined by using Fletcher-Reeves conjugate gradient method as the following: 


$$
\boldsymbol{d}_{k}= \begin{cases}-\boldsymbol{g}_{k} & (k=0) \\ -\boldsymbol{g}_{k}+\frac{\boldsymbol{g}_{k} \cdot \boldsymbol{g}_{k}}{\boldsymbol{g}_{k-1} \cdot \boldsymbol{g}_{k-1}} \mathbf{d}_{k-1} & (k \geq 1)\end{cases}
$$

where $\boldsymbol{g}_{\boldsymbol{k}}$ denotes the vector of reduced gradient of the Lagrangian. The continuous form of the reduced gradient can be taken as the left-hand-side terms of Eq. (9a) with $g=0$ in the regular domain as

$$
\nabla_{\epsilon} \mathcal{L}=-R_{\epsilon} \frac{\partial^{2} \epsilon}{\partial z^{2}}+\int_{0}^{T}\left(\lambda_{E_{x}} \frac{\partial^{2} E_{x}}{\partial t^{2}}\right) d t
$$

If TV regularization scheme were used, the reduced gradient would be

$$
\nabla_{\epsilon} \mathcal{L}=-R_{\epsilon} \beta \frac{\partial^{2} \epsilon}{\partial z^{2}}\left[\left(\frac{d \epsilon}{d z}\right)^{2}+\epsilon\right]^{-\frac{3}{2}}+\int_{0}^{T}\left(\lambda_{E_{x}} \frac{\partial^{2} E_{x}}{\partial t^{2}}\right) d t
$$

In updating the vector $\boldsymbol{\epsilon}_{\boldsymbol{k}}$ by Eq. (11), an inexact line search method was used, where the following Armijo condition was imposed.

$$
J\left(\boldsymbol{\epsilon}_{k}+\alpha \boldsymbol{d}_{k}\right) \leq J\left(\boldsymbol{\epsilon}_{k}\right)+\mu \alpha \boldsymbol{g}_{k} \cdot \mathbf{d}_{k}
$$

In Eq. (15), $\mu$ is usually chosen to be small. $\mu=10^{-10}$ was used herein.

\section{NUMERICAL RESULTS}

Let us consider a one-dimensional PML-truncated domain with the regular domain occupying $-0.1 \mathrm{~m} \leq z \leq 20 \mathrm{~m}$ and the PML placed at $-5.1 \mathrm{~m} \leq z \leq-0.1 \mathrm{~m}$ as well as at $20 \mathrm{~m} \leq z \leq 25 \mathrm{~m}$, as shown in Fig. 2. As target permittivity profiles for reconstruction, two profiles were considered. The first one is a smoothly-varying profile represented by the following function:

$$
\epsilon(z)=\epsilon_{A i r} \times \exp \left[-\frac{(t-10)^{2}}{10}\right]+\epsilon_{A i r}
$$

where $\epsilon_{\text {Air }}$ denotes the permittivity of air and was taken as $\varepsilon_{\text {Air }}=8.847 \times 10^{-12} \mathrm{~F} / \mathrm{m}$. Fig. 3(a) shows the smoothly-varying profile corresponding to Eq. (16). The second profile is a sharply-varying profile as shown in Fig. 3(b), where it is assumed that concrete layer with $\epsilon_{\text {conc }}=4.5 \epsilon_{\text {Air }}$ exists in the range of $8.95 \mathrm{~m} \leq \mathrm{z} \leq 10.95 \mathrm{~m}$.

In this setting, plane electromagnetic waves were assumed to be generated at origin $(z=0)$ by the acceleration of charges $(\partial J / \partial t)$. As the source for electromagnetic waves, a wide-band Gaussian pulse type signal of the following expression was considered:

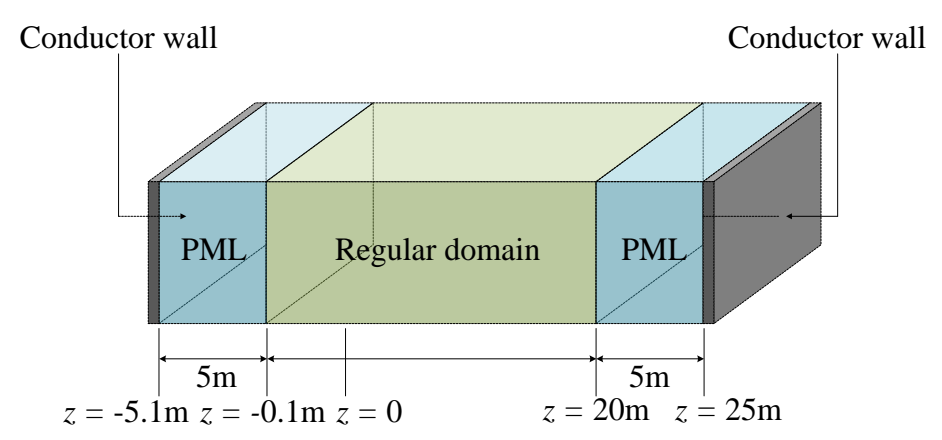

Figure 2: One-dimensional PML-truncated domain for material profile reconstruction 


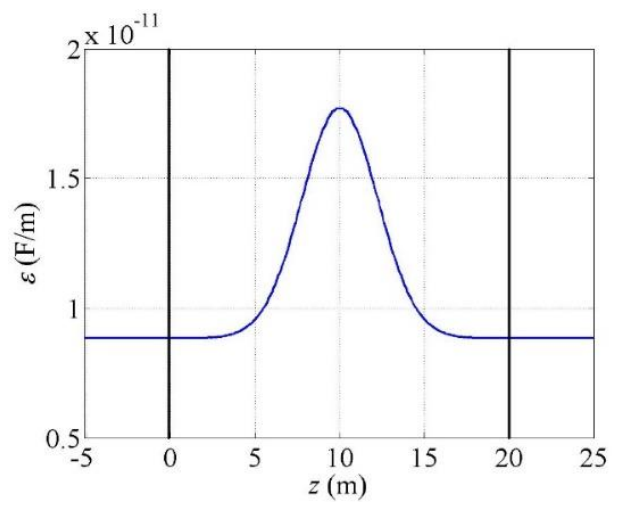

(a) Smooth profile

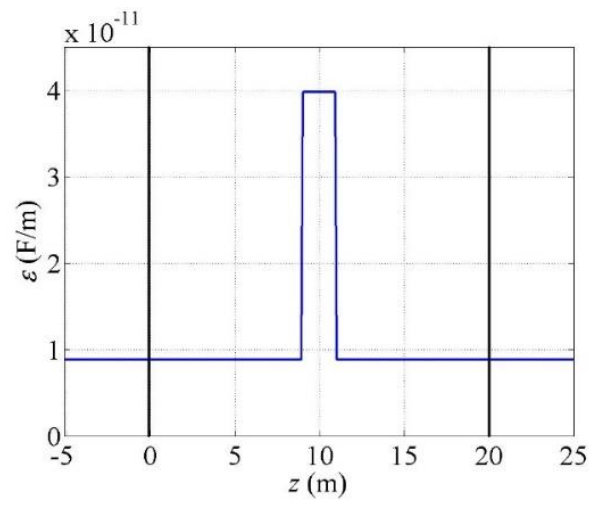

(b) Sharply-varying layered profile

Figure 3: Target permittivity profiles

$$
\frac{\partial J}{\partial t}=10^{13} \exp \left[-\frac{\left(t-5 \times 10^{-8}\right)^{2}}{2 \times 10^{-19}}\right]
$$

Fig. 4 shows the time history and frequency spectrum of the Gaussian pulse, whose maximum frequency is about $2 \mathrm{GHz}$. In the simulation of wave propagation by finite element method, time step was set as $\Delta t=8 \times 10^{-11} \mathrm{~s}$ and element length was set as $L_{e}=0.1 \mathrm{~m}$. Electric field $E_{x}(z, t)$ and magnetic field $H_{y}(z, t)$ were spatially approximated with linear and quadratic shape functions, respectively.

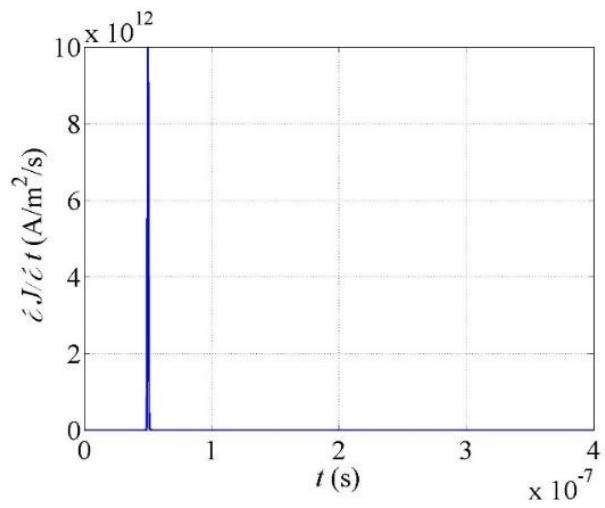

(a) Time history

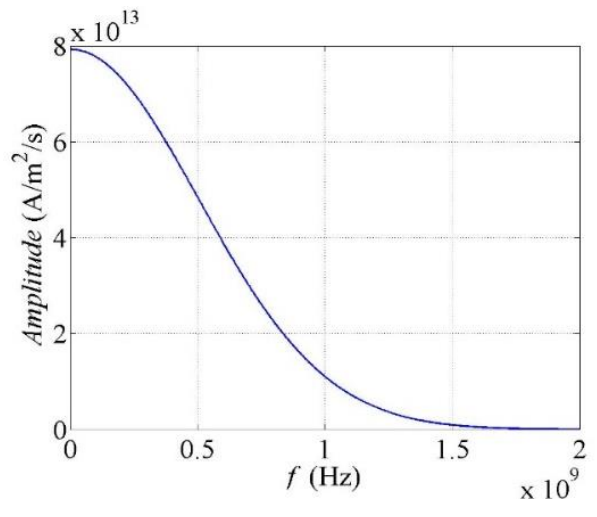

(b) Frequency spectrum

Figure 4: Time history and frequency spectrum of microwave source signal

Fig. 5 shows the smoothly-varying permittivity profiles reconstructed using TN and TV regularization schemes. The inversion started with homogeneous initial guess of $\epsilon(z)=$ $0.75 \times 10^{-11} \mathrm{~F} / \mathrm{m}$, and the target profile was recovered at about 500 iterations with both TN and TV regularizations. Regularization factor was set as $r_{\epsilon}=10^{23}$ for both regularization cases, and the small auxiliary parameter $\beta=10^{-54}$ was used for TV regularization. As can be seen in Fig. 5, both TN and TV regularization schemes assisted the inversion process excellently in capturing the smoothly-varying target profile. Fig. 6 shows the sharply-varying permittivity profiles reconstructed using TN and TV regularization schemes. Initial guess, regularization factor, and the parameter $\beta$ used for TV regularization were the same as in the case of smooth profile inversion. The layered target profile was recovered at about 2,000 iterations for both cases of $\mathrm{TN}$ and $\mathrm{TV}$ regularizations. The inversion with $\mathrm{TV}$ regularization 
reconstructed the sharply-varying material interfaces better than the inversion with TN regularization, but slight oscillations appeared in the recovered profile when TV scheme was used.

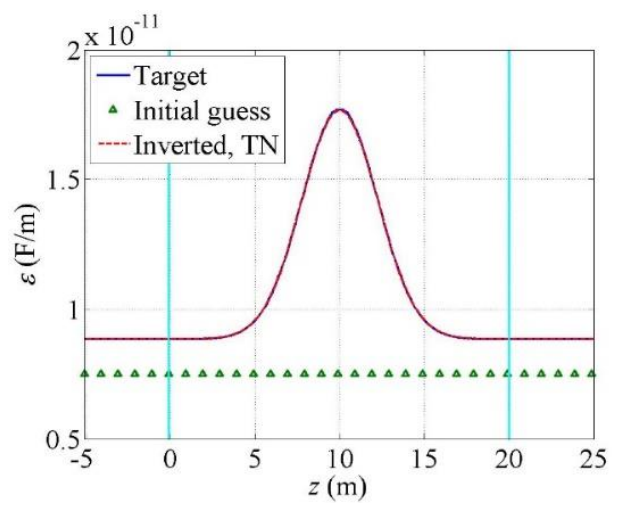

(a) $\mathrm{TN}$ regularization

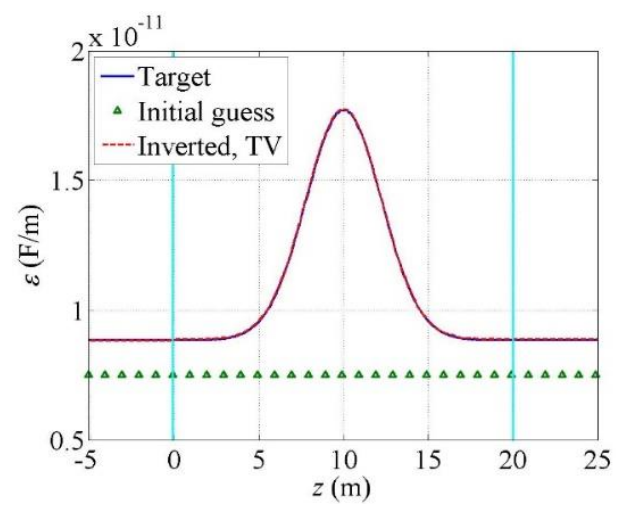

(b) TV regularization

Figure 5: Inverted smoothly-varying permittivity profiles

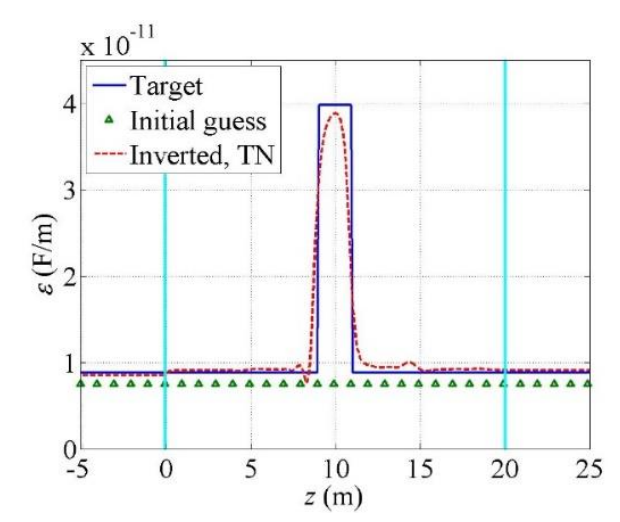

(a) TN regularization

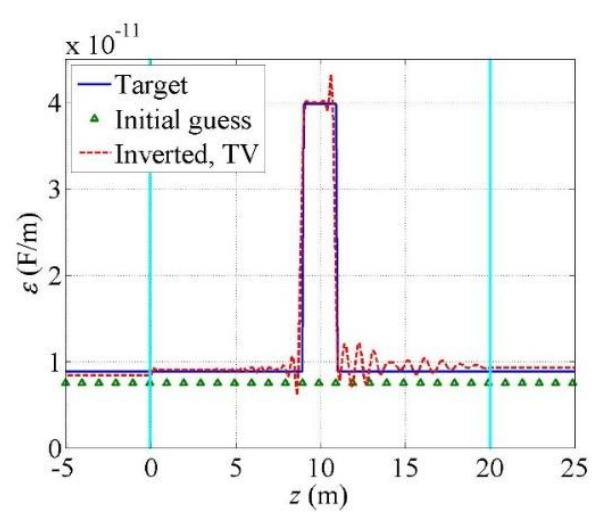

(b) TV regularization

Figure 6: Inverted sharply-varying permittivity profiles

\section{CONCLUSIONS}

- A microwave full-waveform inversion method was developed for the reconstruction of permittivity profile in PML-truncated domains.

- The inversion of microwaves was conducted in a PDE-constrained optimization framework, using PML-endowed Maxwell's equations for plane electromagnetic waves as constraint conditions.

- The permittivity profile of medium was updated iteratively satisfying the first-order optimality conditions of augmented Lagrangian.

- Both smooth and layered permittivity profiles were reconstructed successfully by the fullwaveform inversion method with the aid of Tikhonov and Total Variation regularization schemes. Sharply-varying layered profile could be effectively reconstructed using the Total Variation regularization. 


\section{REFERENCES}

[1] P. Feng, X.Z. Lu, L.P. Ye, Experimental research and finite element analysis of square concrete columns confined by FRP sheets under uniaxial compression. Proceedings of 17th Australasian conference on the mechanics of structures and materials, Gold Coast, Australia, 2002.

[2] I. L. Al-Qadi, D.K. Ghodgaonkar, V.K. Varadan, Effect of moisture on asphaltic concrete at microwave frequencies. IEEE Transactions on Geoscience and Remote Sensing, 29(5), 710-717, 1991.

[3] D.K. Ghodgaonkar, Majid, B.W.A. Wan Mahmood, Majid, B.A. Rosnoizam, Accurate Measurement of Electromagnetic Properties of Concrete for nondestructive Evaluation at Microwave Frequencies. Creating with Concrete, Universiiy of Dundee International Congress, Dundee, Scotland, UK, September 6-10, 672-679, 1999.

[4] H.C. Rhim, O. Büyüköztürk, Electromagnetic properties of concrete at microwave frequency range. Materials Journal, 95(3), 262-271, 1998.

[5] H.C. Rhim, O. Büyüköztürk, Wideband microwave imaging of concrete for nondestructive testing. Journal of Structural Engineering, 126(12), 1451-1457, 2000.

[6] M. Donelli, A. Massa, Computational approach based on a particle swarm optimizer for microwave imaging of two-dimensional dielectric scatterers. Microwave Theory and Techniques, IEEE Transactions on, 53(5), 1761-1776, 2005

[7] M. Benedetti, M. Donelli, A. Martini, M. Pastorino, A. Rosani, A. Massa, An innovative microwave-imaging technique for nondestructive evaluation: Applications to civil structures monitoring and biological bodies inspection. . Instrumentation and Measurement, IEEE Transactions on, 55(6), 1878-1884, 2006

[8] R.G. Pratt, R.M. Shipp, Seismic waveform inversion in the frequency domain, Part 2: Fault delineation in sediments using crosshole data. Geophysics, 64(3), 902-914, 1999.

[9] S. Operto, C. Ravaut, L. Improta, J. Virieux, A. Herrero, P. Dell'Aversana, Quantitative imaging of complex structures from dense wide-aperture seismic data by multiscale traveltime and waveform inversions: a case study. Geophysical prospecting, 52(6), 625$651,2004$.

[10] J.W. Kang, L.F. Kallivokas, The Inverse Medium Problem in 1D PML-truncated Heterogeneous Semi-infinite Domains. Inverse Problems in Science and Engineering, 18(6), 759-786, 2010.

[11] J.W. Kang, L.F. Kallivokas, Mixed Unsplit-field Perfectly-matched-layers for Transient Simulations of Scalar Waves in Heterogeneous Domains. Computational Geosciences, 14(4), 623-648, 2010.

[12] J.W. Kang, L.F. Kallivokas, The Inverse Medium Problem in Heterogeneous PMLtruncated Domains Using Scalar Probing Waves. Computer Methods in Applied Mechanics and Engineering, 200(1-4), 265-283, 2011. 